\title{
Prevalencia de caries de los escolares de la Institución Educativa Rural El Filo de Damaquiel, municipio de San Juan de Urabá, Colombia, 2014
}

\author{
Dayana Ramos-Sanes, Est., OD 1 , Laura Natalia Martínez-Zapata, Est., OD , \\ Esteban Chica-Corrales, Est., OD 1 , Yubisa Patricia Ortiz-Camargo, Est., OD, \\ María Victoria Díaz-Garavito*, MSc
}

Universidad Cooperativa de Colombia, Medellín, Colombia

Recibido: 1 de octubre del 2015 Aprobado: 27 de octubre del 2015

*Autor de correspondencia: María Victoria Díaz Garavito. Facultad de Odontología de la Universidad Cooperativa de Colombia sede Medellín, Carrera 42 n. ${ }^{\circ}$ 49-95, Bloque 8, Medellín, Colombia. Teléfono: (57) 4 4446065. Correo electrónico:

maria.diazg@campusucc.edu.co

Cómo citar este artículo: Ramos-Sanes D, Martínez-Zapata LN, Chica-Corrales E, Ortiz-Camargo YP, Díaz-Garavito MV.

Prevalencia de caries de los escolares de la Institución Educativa Rural El Filo de Damaquiel, municipio de San Juan de Urabá, Colombia, 2014. Rev Nac Odontol. 2016;12(23):49-56. doi: 10.16925/od.v12i23.1380

Resumen. Introducción: la salud bucal forma parte de la salud general y está afectada por determinantes sociales, culturales, educativos, económicos y políticos, de modo que es diferencial entre grupos poblacionales. Objetivo: conocer el perfil bucodental de los niños de la Institución Educativa Rural El Filo de Damaquiel (IER Damaquiel) del corregimiento de San Nicolás en el municipio de San Juan de Urabá (Colombia). Materiales y métodos: se hizo un estudio descriptivo para conocer la prevalencia de caries según el sistema dos dígitos de ICDAs, índice de placa blanda, a 62 escolares entre 4 y 14 años, y se hizo una encuesta sobre prácticas del cuidado bucal a los padres de familia. Se desarrollaron actividades de educación en salud bucal dirigidas a escolares y a padres de familia. Resultados: los niños presentaron una prevalencia de caries de $67,74 \%$, la calidad del cepillado es deficiente en el $82,2 \%$ de los escolares y el consumo de dulces es alto (59,68\% los comen todos los días de la semana). Conclusión: los niños de la IER Damaquiel tienen unos hábitos de cuidado bucal que los enfrenta a factores de riesgo para la salud bucal y, por ende, para su salud general.

Palabras clave: caries dental, ICDAs, índice de placa, prevalencia. 


\title{
Cavities Prevalence in School Children from the Rural Educational Institution El Filo de Damaquiel in the Municipality of San Juan de Uraba, Colombia, 2014
}

\begin{abstract}
Introduction: Healthcare is an important part of general health and can be affected by social, cultural, educational, economic and political determinants, in a way that it is differential among population groups. Objective: To determine the oral profile of the children of the Rural Educational Institute El Filo de Damaquiel (IER Damaquiel) from the small town of San Nicolas in the municipality of San Juan de Uraba (Colombia). Materials and Methods: A descriptive study was performed to determine the prevalence of tooth decay according to the two-digit system of ICDES and index of soft plaques in 62 students between 4 and 14 years of age, and a survey on oral care practices was performed among heads of family. Education activities were developed on oral care addressed to students and heads of family. Results: The children presented a tooth decay prevalence of $67.74 \%$, the tooth brushing quality is inadequate in $82.2 \%$ of students and the intake of sweets is high (59.68\% of the children eat sweets every day of the week). Conclusion: The students of the IER Damaquiel have oral health habits that direct them to risk factors for oral hygiene and therefore to their general health.
\end{abstract}

Keywords: tooth decay, ICDAS, plaque index, prevalence.

\section{Prevalência de cáries dos escolares da Instituição Educativa Rural El Filo de Damaquiel, município de San Juan de Urabá, Colômbia, 2014}

Resumo. Introdução: a saúde bucal faz parte da saúde geral e está afetada por determinantes sociais, culturais, educacionais, económicos e políticos, de modo que é diferencial entre grupos populacionais. Objetivo: conhecer o perfil bucodentário das crianças da Instituição Educativa Rural El Filo de Damaquiel (IER Damaquiel) do bairro de San Nicolás no município de San Juan de Urabá (Colômbia). Materiais e métodos: realizou-se um estudo descritivo para conhecer a prevalência de cáries segundo o sistema dos dígitos de ICDAs, índice de placa branda, a 62 escolares entre 4 e 14 anos, e realizou-se uma pesquisa sobre práticas de higiene bucal aos pais de família. Desenvolveram-se atividades de educação em saúde bucal para os escolares e pais de família. Resultados: as crianças apresentaram uma prevalência de cáries de $67,74 \%$, a qualidade da escovação é deficiente em $82,2 \%$ dos escolares e o consumo de doces é alto (59,68\% comem doces todos os dias da semana). Conclusão: as crianças da IER Damaquiel têm hábitos de cuidado bucal que representam fatores de risco para a saúde bucal e, por tanto, para sua saúde geral.

Palavras chave: cárie dentária, ICDAs, índice de placa, prevalência. 


\section{Introducción}

La salud bucal, al ser parte de la salud general, puede estar afectada por enfermedades infecciosas y crónicas que muestran síntomas en la boca; y por enfermedades bucales que pueden conducir a infecciones e inflamaciones que afectan la salud general [1]. La salud bucal está afectada por determinantes sociales, culturales, educativos, económicos y políticos, de manera que hay diferencias entre grupos poblacionales. Es así como la prevalencia de las enfermedades bucales es mayor en algunas regiones del mundo, donde la pobreza afecta a más población y a los sectores más vulnerables $[2,3,4]$. Desde esta perspectiva, se debe reconocer que hay enfermedades prevenibles, pero esto depende del lugar donde se esté.

Según reportes de la Organización Mundial de la Salud (oms), las enfermedades bucodentales, como la caries dental, la periodontitis (enfermedad gingival) y ahora los cánceres de boca y faringe, son un problema de salud a nivel mundial; sin embargo, los países más afectados son aquellos en vía de desarrollo y, en ellos, las comunidades más pobres. En los países industrializados, la caries dental sigue siendo un problema de salud, afectando al 60-90\% de los escolares y a la mayoría de los adultos [5].

La presencia de las enfermedades bucales es causa de inasistencia escolar y laboral, además de tener altos impactos en la calidad de vida si no se interviene a tiempo. En la población de edad escolar es aún más significativo, pues si no se atiende, puede conducir a daños irreversibles y a problemas de salud general, e incidir en la baja autoestima. A su vez, una mala experiencia dental durante la infancia tiende a producir en los niños una fobia a la consulta odontológica [6].

El informe de la oms resalta la reducción de caries en muchos países desarrollados como resultado de medidas de salud pública, cambio de las condiciones de vida y mejores prácticas de autocuidado [5]. Sin embargo, es de destacar que la caries dental es una enfermedad no erradicada, pero sí controlable, de manera que se debe insistir en las políticas de promoción de la salud y prevención específica.

Aun con la implementación de estrategias preventivas eficaces, el progreso hacia la reducción de caries en la infancia en varias regiones del mundo ha sido poco en los últimos años, lo cual se explica por su relación con las condiciones socioeconómicas, culturales, educativas y de los servicios de salud bucal disponibles [7-9].

En Colombia se han hecho varios estudios nacionales para observar la salud bucal de las poblaciones. Es el caso de la Investigación Nacional de Morbilidad Oral, realizada entre 1965 y 1966 por el Ministerio de Salud y Ascofame; del Estudio Nacional de Salud que incluyó el in Estudio de Morbilidad Oral, en el que se describió el comportamiento de las patologías orales en la población colombiana; del III Estudio Nacional de Salud Bucal (ENSAB); y del in Estudio de factores de riesgo de las enfermedades crónicas en 1998, cuyos resultados mostraron que para la dentición temporal a los cinco años, hubo una reducción del $30 \%$ en el promedio de dientes con historia de caries y cómo el ceo-d pasó de 4,2 en 1977-1980 a 3,0 en 1998, y el historial de caries en los niños a los 12 años pasó de $82,6 \%$ en $1977-1980$ a $71,9 \%$ en 1998 [10].

De igual manera, el último Estudio Nacional de Salud Bucal, realizado en el 2014, concluye que la caries dental sigue siendo la de mayor presencia en toda la población, desde las edades más tempranas hasta las edades adultas; $y$ aunque da cuenta de que se ha mejorado en la atención de la caries, aún se dan caries sin tratar en el 33,84\% de los niños de 1 a 5 años [11].

Colombia es un país con conflictos internos de violencia y discriminación, y aunque resulta imposible elaborar un cuadro completo de la relación entre la violencia y la salud en Colombia, como concluye Franco [12], el hecho de que la violencia cause una marginación de las poblaciones, y entre ellas a las más vulnerables, hace que tenga incidencia en su salud y bienestar. Los altos niveles de violencia significan para el país años de vida saludable perdidos generalmente en población joven en plena edad productiva, e implican altos costos en atención médica por las secuelas físicas y psíquicas de las poblaciones que las sufren [13].

San Juan de Urabá es un municipio colombiano, localizado en la subregión de Urabá, en el norte del departamento de Antioquia (figura 1); cuenta con seis corregimientos y doce veredas, entre ellas El Filo de Damaquiel, donde estála Institución Educativa Rural El Filo de Damaquiel. Allí estudian 130 escolares desde preescolar hasta quinto de primaria; es una comunidad con difícil acceso a los servicios de salud de la zona, debido a su ubicación geográfica, además de ser una región que no es ajena al conflicto armado. 


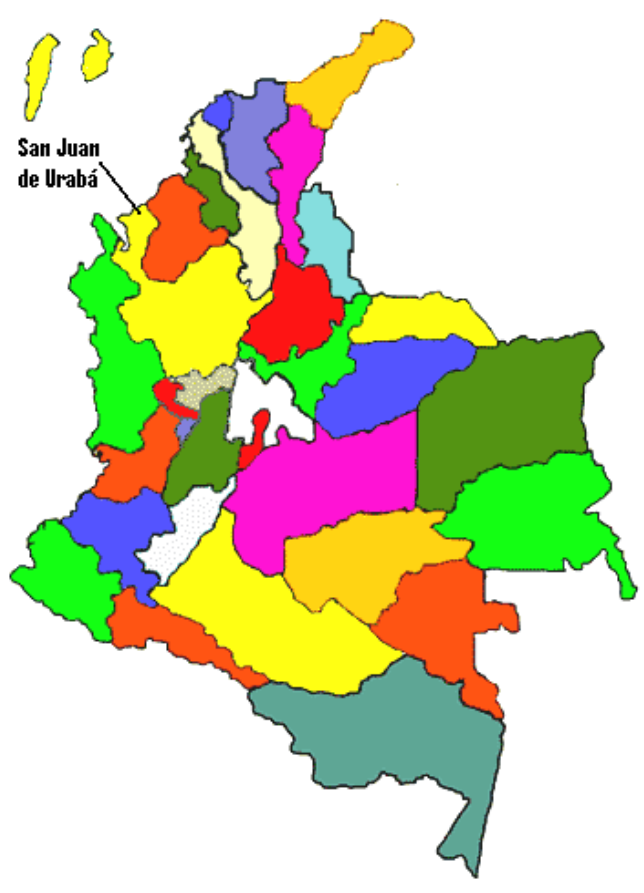

Figura 1. Municipio de San Juan de Urabá

Fuente: http://www.sanjuandeurabaantioquia.gov.co/index.shtml\#2

Las veredas del corregimiento de San Nicolás tienen una población de 1682 habitantes y acceso vía terrestre desde San Juan de Urabá. La principal actividad económica es la agricultura [14].

La red asistencial en salud cuenta con una IPS privada y con la ESE Hospital Héctor Abad Gómez. En su Plan de Desarrollo se da cuenta de la inexistencia de infraestructura y cobertura en la prestación de servicio de salud en las veredas, de la carencia de vías terciarias y el mal estado de las ya existentes, de la falta del servicio de agua potable y de la deficiente cobertura en programas de infancia, adolescencia, juventud, tercera edad, convivencia familiar y organización comunitaria [14].

El interés del estudio es conocer la prevalencia de caries en los escolares de la IER Damaquiel, con el propósito de implementar estrategias de educación en salud y prevención específica.

\section{Materiales y métodos}

Se hizo un estudio descriptivo para conocer la prevalencia de caries dental y los factores de riesgo asociados a ella, en una población de 130 niños para la cual se consideró una prevalencia de caries de
$54,8 \%$ con un error de $5 \%$ y una confianza del $95 \%$, arrojando una muestra de 62 escolares de primero a quinto grado de primaria de la IER Damaquiel.

La selección de la muestra fue aleatoria sistemática y se incluyeron en el estudio los niños cuyos padres firmaron el consentimiento informado, además de contar con la aprobación de los menores para su examen clínico. El estudio se clasificó con riesgo mínimo de acuerdo con el artículo 11b de la Resolución 8430 de 1993 del Ministerio de Salud, y contó con la aprobación del Comité de Ética de la Universidad Cooperativa de Colombia.

Previo a la intervención, los investigadores se estandarizaron en el sistema ICDAs y en el índice de placa bacteriana según Silness y Loe para el examen clínico de los niños. Se hizo el examen clínico, en el que se observó la prevalencia de caries y el índice de placa bacteriana, y se hizo la encuesta sobre saberes y prácticas en salud a los padres de familia de los escolares.

Se creó una base de datos en Microsoft Office Exce ${ }^{\circledR}$ para diligenciar la información, y luego se analizaron las variables de interés de acuerdo con las medidas utilizadas en los estudios descriptivos en el programa estadístico spss 21.0; los resultados se presentaron en tablas de frecuencias y gráficas.

\section{Resultados}

El estudio incluyó a escolares en edades entre 4 y 14 años, siendo 53,2\% niñas. Los escolares de este estudio mostraron una prevalencia modificada C (2-6 ICDAs) op, del 64,51\%, dado que se consideraron los estadios incipientes y avanzados de la caries; sin embargo, al considerar la prevalencia de caries, como lo define el ENSAB IV, solo la caries avanzada se presenta en el 16,13\% de los escolares.

Al observar la severidad de la caries, se encontró que el promedio de dientes con caries moderada fue el más alto con 1,4 dientes, seguida de la caries leve con 1,32 dientes. En las edades de 4 y 5 años, se observó una prevalencia del $16,6 \%$, que aumentó al 66,6\% al considerar las lesiones incipientes de caries, y en la edad de 12 años se observó una prevalencia modificada del $33,3 \%$ teniendo en cuenta las lesiones incipientes.

Respecto a la severidad de la caries por número de dientes, llama la atención que el 22,6\% de los niños presentó caries leve en dos dientes y el 14,5\% en cuatro dientes, seguida de la caries moderada: el $14,5 \%$ de los niños la presentó en cuatro dientes. Es 
decir que la caries incipiente es la que aporta más al indicador de prevalencia.

Al observar la calidad del cepillado, el índice de placa bacteriana de Silness y Loe mostró que el 82,2\% de los escolares tenía un cepillado deficiente; pero al observarlo en relación con la presencia de caries, se puede ver que quienes tuvieron un cepillado deficiente presentaron más caries (tabla 1 y figura 2).

Tabla 1. Calidad del cepillado en relación con la frecuencia del cepillado

\begin{tabular}{|l|c|c|c|c|c|c|}
\hline \multicolumn{4}{|c|}{ Calidad de cepillado (\%) } & \multicolumn{3}{c|}{ Frecuencia de cepillado (\%) } \\
\hline & Buena & Regular & Deficiente & 1 vez & 2 veces & 3 veces \\
\hline Sin presencia de caries & 50 & 55,6 & 31,4 & 45,5 & 31,4 & 33,3 \\
\hline Con presencia de caries & 50 & 44,4 & $\mathbf{6 8 , 6}$ & 54,5 & $\mathbf{6 8 , 6}$ & $\mathbf{6 6 , 7}$ \\
\hline
\end{tabular}

Fuente: elaboración propia

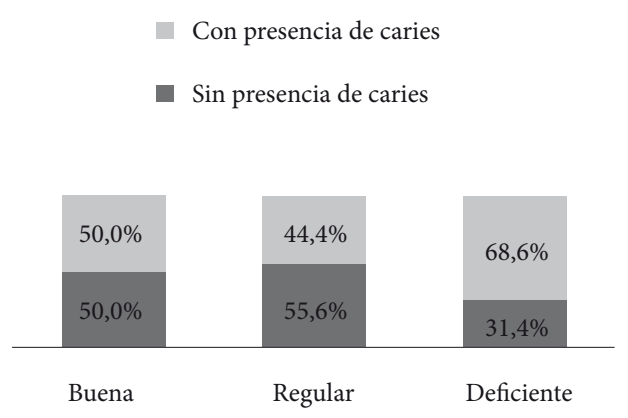

Figura 2. Calidad del cepillado y presencia de caries Fuente: elaboración propia

Al preguntar a los padres de familia sobre la frecuencia de cepillado de sus hijos, respondieron: el $56,45 \%$ lo hace dos veces al día, el $24,19 \%$ tres veces y el 17,7\% una sola vez; sin embargo, al comparar esta frecuencia con la presencia de caries, llama la atención que fue mayor en los que dicen cepillarse más veces al día.

El 95\% usa la crema dental y el cepillo, y solo el $6,4 \%$ usa la seda dental, además de nombrar otros elementos para el cepillado en menor frecuencia, como lo muestra la tabla 2. Al relacionar aquellos que decían usar crema dental y cepillo con la presencia o no de caries, se observó que de los 57 que se cepillan, 36 tienen caries, de manera que la calidad del cepillado, más otros factores, está afectando este resultado.

Los padres consideran que el cuidado de la salud bucal de sus hijos se debe principalmente al uso del cepillado con crema dental (75,80\%), al consumo de leche que protege los dientes $(24,2 \%)$ y al consumo de verduras (8\%).

Sin embargo, al indagar sobre el consumo de azúcares en la dieta de los niños, responden que sus
Tabla 2. Elementos que usan los padres de familia para el cuidado de los dientes de los niños

\begin{tabular}{|l|c|c|}
\hline & N. $^{\circ}$ & $\%$ \\
\hline Cepillo & 59 & 95,16 \\
\hline Crema dental & 60 & 96,77 \\
\hline Seda dental & 4 & 6,45 \\
\hline Enjuagues bucales & 4 & 6,45 \\
\hline Palillos & 6 & 9,67 \\
\hline Bicarbonato & 2 & 3,22 \\
\hline Crema y bicarbonato & 9 & 14,51 \\
\hline
\end{tabular}

Fuente: elaboración propia

hijos consumen dulces todos los días de la semana (60\%) y tres días a la semana (18\%). Al relacionarlo con la presencia o no de caries, se observa una mayor frecuencia de niños con caries entre quienes consumen más dulces a la semana (tabla 3).

Tabla 3. Relación entre consumo de azúcar y presencia de caries en los escolares

\begin{tabular}{|l|c|c|c|}
\hline & $\begin{array}{c}\text { Sin presencia } \\
\text { de caries }\end{array}$ & $\begin{array}{c}\text { Con presencia } \\
\text { de caries }\end{array}$ & Totales \\
\hline Un día & 2 & 3 & 5 \\
\hline Dos días & 2 & 7 & 9 \\
\hline Tres días & 1 & 10 & 11 \\
\hline Todos los días & 17 & 20 & 37 \\
\hline Totales & 22 & 40 & 62 \\
\hline
\end{tabular}

Fuente: elaboración propia

A la par que se hizo la fase de diagnóstico, se desarrollaron actividades de prevención específica 
a los niños, se les entregaron kits de higiene bucal y se dictaron charlas sobre el cuidado de la salud bucal para los padres de familia y los escolares.

\section{Discusión}

El informe sobre el Estado Mundial de la Infancia 2012 confirma que millones de niños están en riesgo de quedar marginados en las grandes y pequeñas ciudades del mundo; además de mostrar que las condiciones de vida de los niños en las zonas urbanas son mejores que las de los niños en las zonas rurales, que generalmente relacionamos con la pobreza y la vulnerabilidad, aunque esto podría estar encubriendo las diferencias existentes entre los niños que residen en las ciudades [15].

A pesar de los esfuerzos y avances en los conocimientos sobre la enfermedad, su prevención y control, se reporta que el problema no ha mejorado en las últimas décadas en los niños con dentición primaria, como sí ha ocurrido en la población en edad escolar [16]. La presencia de caries en la primera infancia se considera como una falla en el sistema de salud y como un reflejo de inequidades en salud en la población, y más cuando están en zonas rurales con menos acceso a los servicios de salud, puesto que constituyen el grupo con mayores necesidades insatisfechas en salud bucal. Un ejemplo de esto es lo reportado por Medina y colaboradores (2006), al observar "que el riesgo de presentar caries en los residentes de la zona conurbana fue mayor que el riesgo de presentar caries en los niños que viven en la zona urbana" $[17,18]$.

Los escolares de este estudio mostraron una prevalencia de caries del $16,12 \%$, que aumentó al $64,52 \%$ al considerar la prevalencia modificada C(2-6 ICDAS) OP; aunque en porcentaje menor, se observa un incremento similar al reportado en el IV ENSAB en Colombia, en el que la prevalencia de caries a los cinco años fue de $52,20 \%$, pero aumentó al observar la prevalencia modificada c(2-6 ICDAs) OP a $81,86 \%$, a expensas principalmente de la detección de los estadios incipientes de caries; y a los doce años pasó de una prevalencia del $37,45 \%$ a $88,49 \%$ al considerar todos los estadios de la caries [7].

En el estudio de Franco y colaboradores (2003) se encuestó a 243 madres de escolares de seis años en Bogotá, Manizales, Medellín y Cartagena, y se encontró que el $95 \%$ considera que el uso del cepillado y la crema dental es la manera de cuidar los dientes de sus hijos, similar a lo reportado por los padres de familia en este estudio $(75,80 \%)$; además, su estudio muestra que el 52,3\% restante cree que son necesarias otras prácticas como la buena alimentación $(65,5 \%)$, visitar al odontólogo (45\%) y evitar el consumo de dulces (10\%) [19]. Sin embargo, en este estudio los encuestados no consideran como parte de su cuidado llevar a los niños al servicio de odontología.

El estudio de Sánchez y colaboradores (2013) en 115 escolares rurales encontró que la prevalencia de caries dental en la dentición temporal fue del $66 \%$, siendo mayor que en los escolares del área urbana; de igual manera, la higiene bucal fue menor en los rurales, con un $69 \%$ con buena higiene y $24 \%$ con higiene regular [20]; a diferencia de este estudio, en el que el $82 \%$ de la muestra presentó una higiene deficiente.

Vargas (2002) observó una prevalencia de caries del $99 \%$ en 100 escolares entre seis y doce años de las comunidades rurales indígenas mapuches de Panguinilague, Puquiñe y Lago Neltume [21], mayor a la reportada en este estudio (64,52\%). De manera similar, Rey (2003), en una población de 357 escolares de seis a catorce años de Moniquirá, Boyacá, encontró una prevalencia de experiencia de caries y de una o más lesiones activas mayor a $97 \%$ [22].

En 1.400 escolares de seis a doce años de edad en León, Nicaragua (2005) [23], se halló que la prevalencia de caries en la dentición temporal a los seis años de edad fue del 72,6\% y en dentición permanente a los doce años fue del 45,0\%; mientras que en la ciudad de Navolato, México (2006), en 3.018 niños de primaria se encontró una prevalencia de caries en la dentición primaria de 90,2\% y en dentición permanente de $82 \%$ [17]. Se puede ver que siguen siendo mayores a la reportada en este estudio.

El estudio realizado en 1.131 escolares de seis a doce años en el municipio de Antolín del Campo en Venezuela (2003) determinó que el $98 \%$ de los entrevistados manifestó utilizar cepillo y crema dental para limpiar sus dientes, y que se cepillan dos o tres veces al día, pero el uso del hilo dental evidenció unas frecuencias menores, mostrando diferencias en las diferentes instituciones, con mayor frecuencia en El Salado (20\%), El Tirano (10\%) y Manzanillo (4,2\%) [24]; se observa que el uso de la seda dental aun es bajo, una apreciación también vista es este estudio que reportó que solo el 6,4\% usa la seda dental. 
Además, el $80 \%$ de los padres de los escolares declaró que sus hijos consumen alimentos que contienen azúcar entre las comidas, los alimentos ingeridos con más frecuencia fueron las chucherías, los refrescos, los jugos, las tortas y los helados [19].

Con respecto al consumo diario de dulces, algunos estudios han encontrado que los niños que ingieren azúcares más de dos veces al día entre comidas tienen 1,3 veces más probabilidad de desarrollar caries. La nutrición y la dieta afectan el desarrollo y la integridad de la cavidad oral, y la progresión de enfermedades de la cavidad oral; interfieren con el equilibrio de la desmineralización de los dientes y la remineralización de varias maneras, pues proporcionan los azúcares y otros carbohidratos fermentables que se metabolizan en ácidos por bacterias de la placa [25]. Aunque los padres de los niños de este estudio dicen que sus hijos consumen dulces de la región, es decir que son artesanales, estos también sufren un proceso de fermentación que disminuye el $\mathrm{pH}$ salival y afecta la estructura del esmalte.

La Academia de Nutrición y Dietética expresa cómo la nutrición es un componente integral de la salud oral y apoya la integración de la salud oral con servicios de nutrición, educación e investigación como una alianza para la promoción de la salud bucal y la prevención de enfermedades y la intervención [26].

\section{Conclusiones}

Se observa una prevalencia de caries alta en la mayoría de los escolares, que se corresponde con un índice de placa deficiente y un alto consumo de dulces a la semana. Llama la atención que pocos padres de familia consideran importante para el cuidado de los dientes del niño, la alimentación con frutas y verduras, el control de consumo de dulces, el uso de la seda dental y llevar al niño a consulta odontológica.

Los niños de la IER Damaquiel tienen una condición de vida que los enfrenta a factores de riesgo para la salud general y la salud bucal, por lo que se deben implementar estrategias de educación en salud e intervención a los factores de riesgo, de acuerdo con las características sociales y culturales.

Se deben implementar estrategias de educación para la salud bucal dirigidas tanto a los niños como a su grupo familiar y a los docentes de la ins- titución, como una manera de evitar los factores que predisponen a la aparición de la caries dental.

\section{Agradecimientos}

A las directivas de la IER Damaquiel, a la familia Martínez Zapata por su hospitalidad, a Colgate Palmolive por su apoyo con los kits de aseo bucal para los escolares, y a los padres de familia y a los niños por su entusiasmo y colaboración. Gracias a la ayuda del profesor Odacir Padilla Sánchez en su aporte al análisis estadístico.

Este artículo presenta los resultados de un proceso investigativo de estudiantes de pregrado de la Facultad de Odontología de la Universidad Cooperativa de Colombia y deja claro que no existen conflictos de autores.

\section{Referencias}

[1] Sheiham A. Oral health, general health and quality of life. Bull World Health Organ. 2005,83(9):644-5. [internet]. Disponible en: http://www.who.int/bulletin/volumes/83/9/644.pdf

[2] Corchuelo J. Sociodemographic differences associated to caries experience and prevalence among users of a public health network. Rev Fac Odontol Univ Antioq. 2012;24(1):96-109.

[3] De la Fuente HJ. Una odontología latinoamericana, iquimera? Revista Odontológica Mexicana. 2007;11(1):53-4 [internet]. Disponible en: http://www. medigraphic.com/pdfs/odon/uo-2007/uo071h.pdf

[4] Agudelo AA, Martínez E. Collective oral health and the Colombian context: a critical analysis. Rev Gerenc Polit Salud. 2009;8(16):91-105.

[5] Petersen PE. The World Oral Health Report 2003. Continuous improvement of oral health in the 21st century-the approach of the wHo Global Oral Health Program. Ginebra: World Health Organization; 2003 [internet]. Disponible en: http://www.who.int/ oral_health/media/en/orh_report03_en.pdf?ua $=1$

[6] World Health Organization. Oral health promotion through schools. wHo Information Series on School Health. Document 11. Ginebra: World Health Organization; 2003 [internet]. Disponible en: http:// www.who.int/oral_health/media/en/orh_school_ doc11.pdf

[7] Hale KJ. Oral health risk assessment timing and establishment of the dental home. Pediatrics. 2003;111(5Pt1):1113-6. 
[8] Mouradian WE, Wehr E, Crall JJ. Disparities in children's oral health and access to dental care. JAMA. 2000;284(20):2625-31.

[9] Petersen PE, Bourgeois D, Ogawa H, Estupinan-Day $S$, Ndiaye C. La carga mundial de enfermedades bucodentales y riesgos para la salud oral. Bull World Health Organ. 2005;83(9):661-9.

[10] Salud Bucal-ensab III. II Estudio Nacional de Factores de Riesgo de Enfermedades Crónicas-EnfreC II. Bogotá: Ministerio de Salud; 1999.

[11] Ministerio de Salud de Colombia. IV Estudio Nacional de Salud Bucal. Para saber cómo estamos y saber qué hacemos. Bogotá: Ministerio de Salud; 2014.

[12] Franco Agudelo S. Violencia y salud en Colombia. Rev Panam Salud Pública. 1997;1(2):93-103.

[13] Ayala ML. La violencia en Colombia: una aproximación desde la salud mental. Cuadernos Hispanoamericanos de Psicología. 2010;2(1):13-36.

[14] Secretaría Seccional de Salud y Protección Social de Antioquia. Plan Territorial de Salud 2008-2011 San Juan de Urabá. Medellín: Secretaría Seccional de Salud y Protección Social de Antioquia; 2008.

[15] Lake A. Informe del Estado Mundial de la Infancia 2012 del Fondo de las Naciones Unidas para la Infancia (Unicef). Nueva York: Unicef; 2012.

[16] Bönecker M, Cleaton-Jones P. Trends in dental caries in Latin American and The Caribbean 5-6- and 11-13-year-old children: a systematic review. Community Dent Oral Epidemiol. 2003;31:152-7.

[17] Villalobos Rodelo JJ, Medina Solís CE, Molina Frechero N, Vallejos Sánchez AA, Pontigo Loyola AP, Espinoza Beltrán JL. Caries dental en escolares de 6 a 12 años de edad en Navolato, Sinaloa, México: experiencia, prevalencia, gravedad y necesidades de tratamiento. Biomédica. 2006;26(2):224-33.

[18] Medina-Solís CE, Maupomé G, Pelcastre-Villafuerte B, Ávila-Burgos L, Vallejos-Sánchez AA, Casanova
-Rosado AJ. Desigualdades socioeconómicas en salud bucal: caries dental en niños de 6 a 12 años de edad. Rev Invest Clín. 2006;58(4):296-304.

[19] Franco AM, Santamaría A, Kurzer E, Castro L, Giraldo M. El menor de seis años: situación de caries y conocimientos y prácticas de cuidado bucal de sus madres. Revista CEs Odontología. 2004;17(1).

[20] Sánchez-Perez L, Alanís-Tavira J, Vera-Hermosillo $\mathrm{H}$, Rodríguez-Gurza ME, Arjona-Serrano J, Sáenz -Martínez LP. Factores de riesgo para caries en escolares rurales y urbanos de Yucatán. Ciencias Clínicas. 2013;14(1):3-11.

[21] Vargas-Aros R, Herrera-Catrileo M. Prevalence study of dental caries in students from Mapuche rural communities of Panguinilague, Puquiñe \& Neltume Lake. Valdivia Province. $\mathrm{x}$ Lakes Region. Revista Dental de Chile. 2002;93(3):3-8

[22] Rey MA, Salas E, Martignon S. Caries dental y asociación a factores de riesgo en la población escolar de Moniquirá, Boyacá. Revista Científica. 2003;9(2):29-34.

[23] Herrera MDS, Medina-Solís CE, Maupomé G. Prevalencia de caries dental en escolares de 6-12 años de edad de León, Nicaragua. Gac Sanit. 2005;19(4):302-6.

[24] Mendes DD, Caricote N. Prevalencia de caries dental en escolares de 6 a 12 años de edad del municipio Antolín del Campo, estado Nueva Esparta, Venezuela (2002-2003). Revista Latinoamericana de Ortodoncia y Odontopediatría. 2003; edición electrónica [internet]. Disponible en: https://www. ortodoncia.ws/publicaciones/2003/art7.asp

[25] Touger-Decker R, Van Loveren C. Sugars and dental caries. Am J Clin Nutr. 2003;78(4):881S-892S.

[26] Touger-Decker R, Mobley C. Position of the Academy of Nutrition and Dietetics: oral health and nutrition. J Nutr Diet Acad. 2013;113(5):693-701. 